\title{
Forschung am Menschen
}

Prof. Peter M. Suter, Genf

Präsident SAMW
Symposium, 31. März 2006, Bern (Kursaal)

\section{Forschung am Menschen:}

\section{Die Diskussion ist eröffnet}

Die medizinisch-ethischen Richtlinien der Schweizerischen Akademie der Medizinischen Wissenschaften (SAMW) über «Forschungsuntersuchungen am Menschen» von 1997 hielten fest, dass wissenschaftlicher Fortschritt nur dann ethisch vertretbar sei, wenn die Respektierung der Menschenwürde aller Personen gewährleistet bleibe. Gleichzeitig wurde konstatiert, dass «die heutige Gesetzgebung für den Bereich Forschungsuntersuchungen am Menschen [...] lückenhaft, unsystematisch und oft schwer aufzufinden [ist]». Im Jahr 2000 hat deshalb das Parlament den Bundesrat beauftragt, ein Gesetz über die Forschung am Menschen auszuarbeiten.

Das Bundesamt für Gesundheit, das bei der Ausarbeitung dieses Gesetzes federführend war, beschreibt seine Aufgabe wie folgt: «Forschung am Menschen wirft ethische und rechtliche Fragen auf. Der in der Verfassung verankerte Persön- lichkeitsschutz und die ebenfalls in der Verfassung verbriefte Forschungsfreiheit können miteinander in Konflikt geraten. Ziel eines Bundesgesetzes über die Forschung am Menschen ist die Schaffung einer umfassenden und ausgeglichenen Regelung, die den Schutz der Versuchspersonen einerseits und die Voraussetzung für neue wissenschaftliche Erkenntnisse andererseits gewährleistet.»

Der Bundesrat hat den Gesetzesentwurf Anfang Februar 2006 veröffentlicht; dieser ist nun Gegenstand einer breiten Vernehmlassung. Das von der SAMW gemeinsam mit SNF, SWTR, Swissmedic, BAG und IDS organisierte Symposium bringt Fachleute aus den Bereichen Medizin, Recht, Wissenschaftspolitik und Ethik aus dem In- und Ausland zusammen; gemeinsam mit den übrigen Teilnehmern/-innen dieser Tagung werden sie prüfen, inwieweit der Gesetzesentwurf den oben formulierten Zielen Rechnung trägt und wo allenfalls Änderungs- bzw. Ergänzungsbedarf besteht.

\section{Programm}

9.30 Begrüssung und Einführung

Prof. Peter M. Suter, Präsident SAMW, Genf

9.40 Ein Gesetz über «Forschung am Menschen»: Was wollen die Behörden? Prof. Thomas Zeltner, Direktor des Bundesamtes für Gesundheit, Bern

10.10 Kein medizinischer Fortschritt ohne Forschung am Menschen Prof. Peter Meier-Abt, Vizerektor Forschung, Universität Basel

10.40 Forschung am Menschen: die sozialwissenschaftliche Perspektive Prof. Ulrike Ehlert, Vorsteherin des Psychologischen Institutes der Universität Zürich

11.10 Kaffeepause

11.30 Consentement chez le sujet de recherche incapable de discernement Prof. François Lemaire, Hôpital Henri-Mondor, Créteil (France)

12.00 The proposed law in Norway: critical perspectives on certain aspects of legal regulation of medical research Prof. Knut Ruyter, National Ethics Committee for Research, Oslo (Norway)

12.30 Mittagessen 


\subsection{Workshops}

I Aufgaben der Ethikkommissionen

Experten: Giovan Maria Zanini, Bellinzona; Fürsprecher Michael Gerber, BAG, Bern Moderation: Prof. Dominique Sprumont, IDS, Neuchâtel

II Schadendeckung

Experten: Dr. Christoph Zenger, Bern; lic. iur. Jean-Christophe Méroz, Swissmedic, Bern Moderation: Prof. Daniel Scheidegger, SNF, Basel

III Besonders schutzbedürftige Versuchspersonen

Experten: Prof. Susanne Suter, Genf; lic. iur. Dolores Krapf, BAG, Bern

Moderation: Paul Dietschy, Swissmedic, Bern

IV Sozialwissenschaftliche Forschung

Experten: Dr. Alain Kaufmann, Lausanne; Dr. Verena Schwander, BAG, Bern

Moderation: Prof. Joseph Jurt, SWTR, Freiburg (D)

$V$ Forschung mit biologischem Material und Personendaten (inkl. Biobanken)

Experten: Prof. Volker Dittmann, Basel; Dr. Andrea Arz de Falco, BAG, Bern

Moderation: Dr. Margrit Leuthold, SAMW, Basel

VI Forschung an verstorbenen Personen

Experten: Prof. Robert Maurer, Zürich; Biol., Pol. Wiss. Katja Manike, BAG, Bern

Moderation: Prof. Stephanie Clarke, SAMW, Lausanne

VII Forschung an fötalem Gewebe

Experten: Prof. Wolfgang Holzgreve, Basel; dipl. biol., lic. iur. Matthias Bürgin, BAG, Bern Moderation: Prof. Alex Mauron, SWTR, Genf

15.00 Kaffeepause

\subsection{Berichte aus den Workshops}

16.45 Podium: Grenzen der gesetzlichen Regelung der Forschung am Menschen Teilnehmer/innen: Prof. Daniel Scheidegger, Basel; Prof. Peter Meier-Abt, Basel; Prof. Ulrike Ehlert, Zürich; Dr. Thomas Cueni, Generalsekretär Interpharma, Basel; Prof. Dominique Sprumont, Institut de droit de la santé, Université de Neuchâtel

\subsection{Ausblick}

Prof. Peter M. Suter, Genf

Anmeldung unter www.samw.ch. 